\title{
Effects of Mass Transfer, Radiation, Joule Heating, and Viscous Dissipation on Steady MHD Marangoni Convection Flow over a Flat Surface with Suction and Injection
}

\author{
S. Mohammed Ibrahim \\ Department of Mathematics, Priyadarshini College of Engineering \& Technology, Nellore 524004, India \\ Correspondence should be addressed to S. Mohammed Ibrahim; ibrahimsvu@gmail.com
}

Received 4 August 2013; Accepted 6 November 2013

Academic Editor: Giuseppe Carbone

Copyright ( $\odot 2013$ S. Mohammed Ibrahim. This is an open access article distributed under the Creative Commons Attribution License, which permits unrestricted use, distribution, and reproduction in any medium, provided the original work is properly cited.

\begin{abstract}
The combined effects of radiation and mass transfer on a steady MHD two-dimensional Marangoni convection flow over a flat surface in presence of Joule heating and viscous dissipation under influence of suction and injection is studied numerically. The general governing partial differential equations are transformed into a set of nonlinear ordinary differential equations by using unique similarity transformation. Numerical solutions of the similarity equations are obtained using the Runge-Kutta method along with shooting technique. The effects of governing parameters on velocity, temperature, and concentration as well as interface velocity, the surface temperature gradient, and the surface concentration gradient were presented in graphical and tabular forms. Comparisons with previously published work are performed and the results are found to be in excellent agreement.
\end{abstract}

\section{Introduction}

In recent years, many researchers have investigated the Marangoni convection and applied such convection to their problems. This is mainly because it is important in crystal growth melts and greatly influences other industrial processes. Nishino and Kawamura [1] stated that Marangoni convection negatively affects the quality of silicon crystals for semiconductors and the convection also occurs in heat pipe for heat radiation devices of personal computers. The surface tension gradient variations along the interface may induce the Marangoni convection. In particular, the surface tension gradients that are responsible for Marangoni convection can be due to gradients of temperature (thermal convection) and/or concentration (solutal convection). A lot of analyses in Marangoni convection have been discovered in various geometries and conditions. Some of experimental works linked to Marangoni convection were discussed in several papers by Arafune and Hirata [2], Arafune et al. [3], Galazka and Wilke [4], Neumann et al. [5], Arendt and Eggers [6], Xu et al. [7], and Christopher and Wang [8].
On the other hand, the study of Magnetohydrodynamics (MHD) is important in the heat and mass transport process. The study of heat transfer is integral part of natural convection flow and belongs to the class of problems in boundary layer theory. The quality of heat transferred is highly dependent upon the fluid motion within the boundary layer. A large number of physical phenomena involve natural convection that was studied by Jaluria [9], which are enhanced and driven by internal heat generation. In such flows the buoyancy force is incremented due to heat generation resulting in modification of heat transfer characteristic. The effect of internal heat generation is especially pronounced for low Prandtl number fluid. MHD is the study of motion of electrically conducting incompressible fluid in the presence of magnetic field, that is, an electromagnetic field interacting with the velocity field of an electrically conducting fluid. Hydromagnetic flows have become important due to industrial applications; for instance it is used to deal with the problem of cooling of nuclear reactor by fluid having very low Prandtl number that was studied by Itaru et al. [10] and Fumizawa [11]. Moreover, Al-Mudhaf and Chamkha [12] investigated the similarity 
solution for MHD thermosolutal Marangoni convection over a flat surface in the presence of heat generation or absorption with fluid suction and injection.

It was realized that the studies of thermal radiation with heat and mass transfer are important in electrical power generation, astrophysical flows, solar power technology, and other industrial areas. Pathak and Maheshwari [13] have analyzed the influence of radiation on an unsteady free convection flow bounded by an oscillating plate with variable wall temperature. The effects of thermal radiation, buoyancy, and suction/blowing on natural convection heat and mass transfer over a semi-infinite stretching surface have been studied by Shateyi [14]. Moreover, Suneetha et al. [15] have investigated the radiation effects on the MHD free convection flow past an impulsively started vertical plate with variable surface temperature and concentration.

The effects of viscous dissipation and Joule heating are usually characterized by the Eckert number and the product of the Eckert number and magnetic parameter, respectively, and both have a very important part in geophysical flow and in nuclear engineering that was studied by Alim et al [16], the effects of suction or injection on boundary layer flow also have a huge influence over the engineering application and have been widely investigated by numerous researchers. With this understanding, many researchers studied the effects of suction or injection in various geometries, for example, Borisevish and Potanin [17] for heat transfer near a rotating disk. Duwairi [18] and Chen [19] for MHD convection flow in the presence of radiation.

However, the study of the combined effects of mass transfer and radiation on steady MHD Marangoni convection flow of a dissipative fluid has received a little attention. Hence, the object of the present paper is to analyze the combined effects of radiation and mass transfer on steady MHD laminar Marangoni convection boundary layer flow of an electrically conducting fluid past a flat surface, by taking Joule heating and viscous dissipation under influence of suction or injection. The governing partial differential equations are reduced to a system of self-similar equations using the similarity transformations. The resultant equations are then solved numerically using the Runge-Kutta fourth order technique along with shooting method. The effects of governing physical parameters on the velocity, temperature, and concentration as well as surface velocity, surface temperature, and surface concentration gradient are computed and presented in graphical and tabular forms. To verify the obtained results, we have compared the present numerical results with previous work by Al-Mudhaf and Chamkha [12]. The comparison results show a good agreement and we are confident that our present numerical results are accurate.

\section{Mathematical Analysis}

A steady, two-dimensional, laminar boundary layer flow of a viscous incompressible electrically conducting and radiating fluid over a flat surface is considered. The fluid is assumed to be gray, absorbing but nonscattering. The $x$-axis is taken along the surface and $y$-axis normal to it. The surface is assumed to be in the presence of the surface tension due to temperature and concentration gradients at the wall. Further, a uniform magnetic field of strength $B_{0}$ is applied normal to the surface which then produces the magnetic forces along the surface. The fluid is assumed to be slightly conducting, and hence the magnetic Reynolds number is much less than unity and the induced magnetic field is negligible in comparison with the applied magnetic field. Under the above assumptions and with the usual boundary layer approximations, the governing equations are [20] as follows.

Continuity equation

$$
\frac{\partial u}{\partial x}+\frac{\partial u}{\partial y}=0
$$

Momentum equation

$$
u \frac{\partial u}{\partial x}+v \frac{\partial u}{\partial y}=v \frac{\partial^{2} u}{\partial y^{2}}-\frac{\Delta B_{0}^{2}}{\rho} u
$$

Energy equation

$$
u \frac{\partial T}{\partial x}+v \frac{\partial T}{\partial y}=\frac{k}{\rho c_{p}} \frac{\partial^{2} T}{\partial y^{2}}-\frac{1}{\rho c_{p}} \frac{\partial q_{r}}{\partial y}+\frac{\Delta B_{0}^{2}}{\rho c_{p}} u^{2}+\frac{\nu}{c_{p}}\left(\frac{\partial u}{\partial y}\right)^{2} .
$$

Species equation

$$
u \frac{\partial C}{\partial x}+v \frac{\partial C}{\partial y}=D \frac{\partial^{2} C}{\partial y^{2}}
$$

The boundary conditions for the velocity, temperature, and concentration fields are

$$
\begin{gathered}
v(x, 0)=-v_{w}, \quad T(x, 0)=T_{\infty}+A x^{2}, \\
C(x, 0)=C_{\infty}+A^{*} x^{2}, \\
\mu \frac{\partial u}{\partial y}=-\frac{d \sigma}{d T} \frac{\partial T}{\partial x}-\frac{d \sigma}{d C} \frac{\partial C}{\partial x} \quad \text { at } y=0 \\
u(x, \infty)=0, \quad T(x, \infty)=T_{\infty}, \\
C(x, \infty)=C_{\infty} \quad \text { as } y \longrightarrow \infty
\end{gathered}
$$

where $u$ and $v$ are the velocity components in the $x$ and $y$ directions, respectively. $v$ is the kinematic viscosity, $\Delta$ is the electric conductivity, $\sigma$ is the surface tension, $B_{0}$ is the uniform magnetic field strength, $\rho$ is the density of the fluid, $c_{p}$ is the specific heat at constant pressure, $T$ is the fluid temperature, $T_{\infty}$ is the fluid temperature far from the surface, $C$ is the species concentration, $C_{\infty}$ is the species concentration far from the surface, $A$ is the temperature gradient coefficient, $A^{*}$ is the concentration gradient coefficient, $\mu$ is the dynamic viscosity, $k$ is the thermal conductivity of the fluid, $q_{r}$ is the radiative heat flux, and $v_{w}$ is constant suction $\left(v_{w}>0\right)$ or injection $\left(v_{w}<0\right)$ velocity. The second, third, and fourth terms on the right hand side of (3) represent the radiation, Joule heating, 
and viscous dissipation, respectively. The fourth condition of (5) represents the Marangonic coupling condition at the interface.

When we use the Rosseland approximation for radiation, the radiative heat flux can be simplified as [21]

$$
q_{r}=-\frac{4 \sigma^{*}}{3 k^{*}} \frac{\partial T^{4}}{\partial y}
$$

where $\sigma^{*}$ is the Stefan-Boltzmann constant and $k^{*}$ is the mean absorption coefficient. It is assumed that the temperature differences within the flow are small, so that the term $T^{4}$ may be expressed as a linear function of temperature. Hence by expanding $T^{4}$ in a Taylor's series about $T_{\infty}$ and neglecting higher-order terms,

$$
T^{4} \approx 4 T_{\infty}^{3} T-3 T_{\infty}^{4}
$$

Using (7) and (8), (3) reduces [22]:

$$
u \frac{\partial T}{\partial x}+v \frac{\partial T}{\partial y}=\alpha(1+\mathrm{Nr}) \frac{\partial^{2} T}{\partial y^{2}}+\frac{\Delta B_{0}^{2}}{\rho c_{p}} u^{2}+\frac{v}{c_{p}}\left(\frac{\partial u}{\partial y}\right)^{2},
$$

where $\alpha=k / \rho c_{p}$ is the thermal diffusivity and $\mathrm{Nr}=$ $16 \sigma^{*} T_{\infty}^{3} / 3 k k^{*}$ is the radiation parameter.

Further, we use the similarity transformation by AlMudhaf and Chamkha [12] and the standard definition of the stream function such that $u=\partial \psi / \partial y$ and $v=-\partial \psi / \partial x$ to obtain the similarity solution of the problems. The similarity transformations are given by

$$
\begin{aligned}
& \eta=C_{1} y, \quad f(\eta)=C_{2} x^{-1} \psi(x, y), \\
& \theta(\eta)=\frac{\left[T(x, y)-T_{\infty}\right]}{A} x^{-2}, \\
& \phi(\eta)=\frac{\left[C(x, y)-C_{\infty}\right]}{A^{*}} x^{-2},
\end{aligned}
$$

where

$$
\begin{aligned}
& A=\frac{\Delta T}{L^{2}}, \quad A^{*}=\frac{\Delta C}{L^{2}}, \\
& C_{1}=\sqrt[3]{\frac{\rho A(d \sigma / d T)}{\mu^{2}}}, \quad C_{2}=\sqrt[3]{\frac{\rho^{2}}{\mu A(d \sigma / d T)}}
\end{aligned}
$$

with $L$ being the length of the surface and $\Delta T$ and $\Delta C$ are the constant characteristics temperature and concentration, respectively. Substituting (10) into (2), (4), and (9), we obtain the following ordinary differential equations:

$$
\begin{gathered}
f^{\prime \prime \prime}+f f^{\prime \prime}-\left(f^{\prime}\right)^{2}-M^{2} f^{\prime}=0, \\
\frac{(1+\mathrm{Nr})}{\operatorname{Pr}} \theta^{\prime \prime}-2 f^{\prime} \theta+f \theta^{\prime}+\operatorname{Ec}\left[M^{2}\left(f^{\prime}\right)^{2}+\left(f^{\prime \prime}\right)^{2}\right]=0 \\
\phi^{\prime \prime}+\operatorname{Sc}\left(f \phi^{\prime}-2 f^{\prime} \phi\right)=0,
\end{gathered}
$$

where primes denote differentiation with respect to $\eta, \operatorname{Pr}=$ $\nu / \alpha$ is the Prandtl number, $M^{2}=\Delta B_{0}^{2} C_{2} / \rho C_{1}$ is the magnetic field parameter, $\mathrm{Ec}=C_{1}^{2} / A c_{p} C_{2}^{2}$ is the Eckert number, Sc $=\nu / D$ is the Schmidt number, $f_{w}(>0)$ is the constant suction parameter, and $f_{w}(<0)$ is the constant injection parameter. It should be mentioned here again that the viscous dissipation effect is examined using the Eckert number Ec, while the product of the Eckert number and the magnetic field parameter $M$ gives the joule heating.

The dimensionless form of the boundary conditions become

$$
\begin{aligned}
& f(0)=f_{w}, \quad f^{\prime \prime}(0)=-2, \quad \theta(0)=1, \quad \phi(0)=1, \\
& f^{\prime}(\infty)=0, \quad \theta(\infty)=0, \quad \phi(\infty)=0 .
\end{aligned}
$$

\section{Solution of the Problem}

The nonlinear ordinary differential equations (12) subjects to the boundary conditions (13) are solved numerically using the Runge-Kutta fourth order technique along with shooting method. In this method, it is most important to choose the appropriate finite value of the edge of boundary layer, $\eta \rightarrow$ $\infty$ (say $\eta_{\infty}$ ), that is between 4 to 8 , which is in accordance with the standard practice in the boundary layer analysis. First of all, higher-order nonlinear differential equations (12) are converted into simultaneous linear differential equations and they are further transformed into initial value problem by applying the shooting method. The resultant initial value problem is solved by employing Runge-Kutta fourth order technique. The initial step size $h=\Delta \eta=0.01$ is used to obtain the numerical solution with five decimal place accuracy as the criterion of convergence.

\section{Results and Discussion}

In order to get a physical insight into the problem, a representative set of numerical results are shown in Figures 1-18 which illustrates the influence of physical parameters namely, the magnetic parameter $M$, Prandtl number Pr, radiation parameter $\mathrm{Nr}$, Eckert number Ec, Schmidt number Sc, Suction, and Injection $f_{w}$ on the velocity $f^{\prime}(\eta)$, temperature $\theta(\eta)$ and concentration $\phi(\eta)$, profiles as well as the reduced velocity at the interface, reduced heat transfer, and reduced mass transfer at the interface. Throughout the calculations, the parametric values are fixed to be $\operatorname{Pr}=0.72, M=1.0$, $\mathrm{Nr}=0.5, \mathrm{Ec}=0.1, \mathrm{Sc}=0.6$, and $f_{w}=1.0$, unless otherwise indicated.

Figures 1, 2, and 3 show the effect of magnetic parameter $M$ in the influence of suction and injection on velocity, temperature, and concentration profiles. Application of a transverse magnetic field results in a drag-like force called the Lorentz force. This force tends to slow down the movement of the fluid along surface and to increase its temperature and the concentration species. This is evident in the decreases in the velocities and increases in the temperature and concentration as $M$ increases. In addition, as the strength of magnetic 


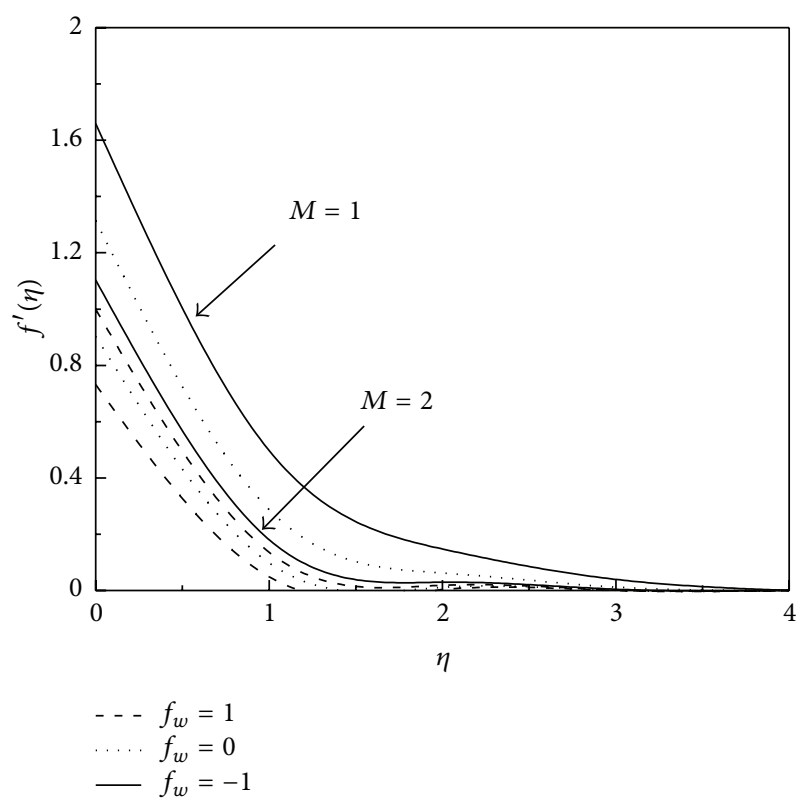

FIGURE 1: Effects of $M$ on velocity profiles with various values of $f_{w}$.

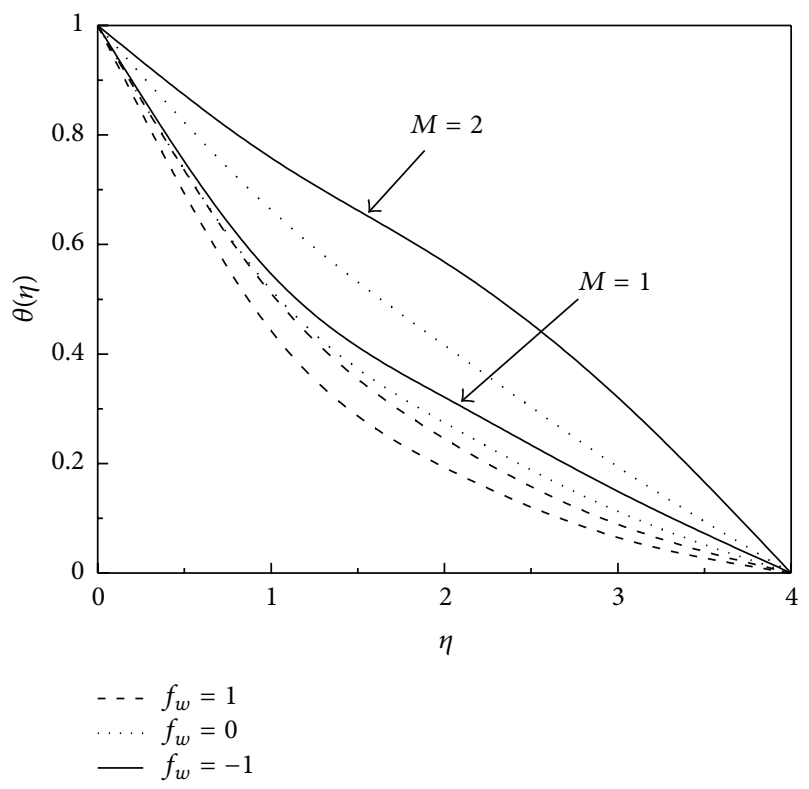

FIGURE 2: Effects of $M$ on temperature profiles with various values of $f_{w}$.

field increases, the hydrodynamic velocity boundary layer decreases while the thermal and solutal (concentration) boundary layer. It is also noticed from Figure 1 that the wall velocity is nonzero due to the Marangoni or surface tension effect and it decreases as $M$ increases. These behaviors are depicted in Figures 1, 2, and 3.

Figure 4 illustrates the effect of Prandtl number Pr in the influence of suction and injection on temperature profile. The result indicates that the increasing of Prandtl number decreases the temperature profile.

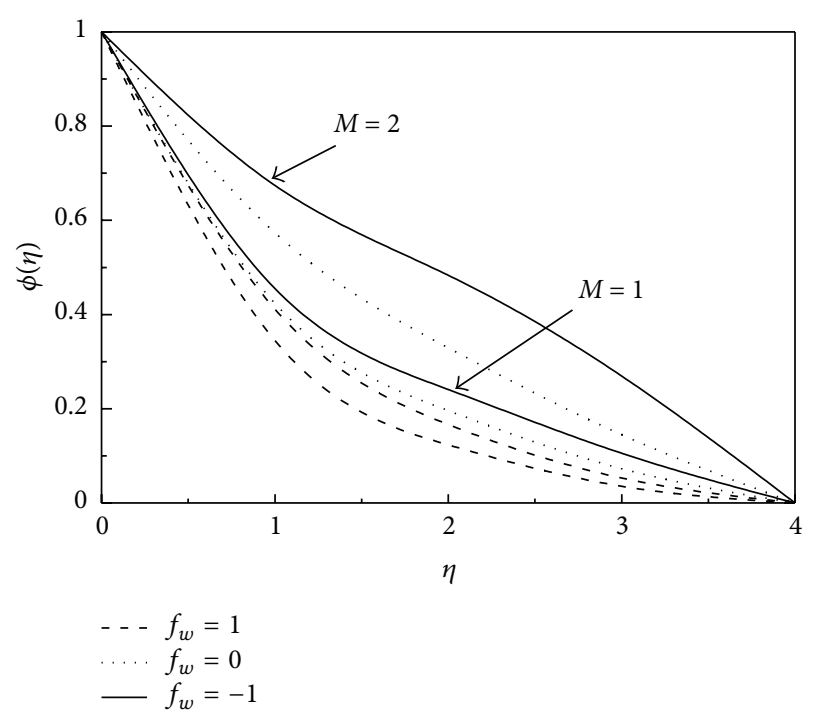

FIGURE 3: Effects of $M$ on concentration profiles with various values of $f_{w}$.

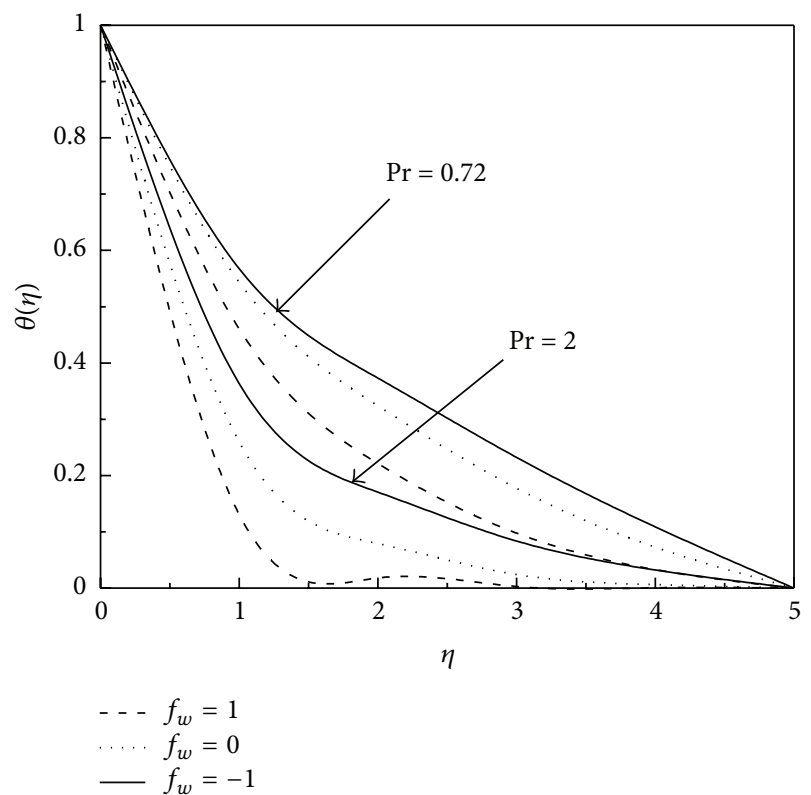

FIGURE 4: Effects of Pr on temperature profiles with various values of $f_{w}$.

Effect of radiation parameter $\mathrm{Nr}$ on temperature of the fluid with the influence of the suction or injection parameter $f_{w}$ is presented in Figures 5, 6, 7, and 8. Figure 5 displays the variation with $\mathrm{Nr}$ of the reduced temperature gradient, $-\theta^{\prime}(0)$, with different values of $f_{w}$. One can see that the reduced temperature gradient, $-\theta^{\prime}(0)$, decreases as $\mathrm{Nr}$ increases. Thus, the heat transfer rate at the surface decreases in the presence of radiation. This result qualitatively agrees with expectations, since the effect of radiation is to decrease the rate of energy transport to the fluid, thereby decreasing the temperature of the fluid. From Figures 6-8, we observe that the temperature profiles increase as radiation parameter 


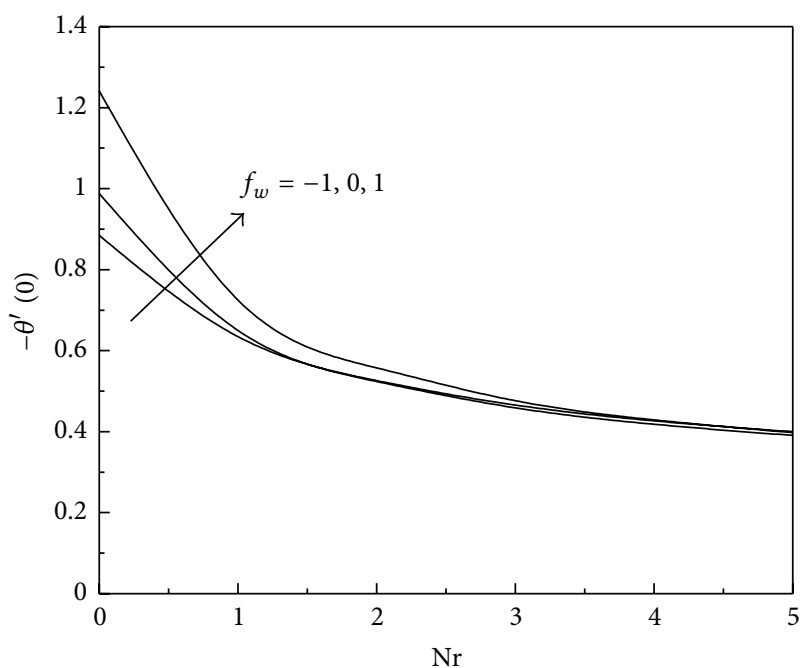

FIGURE 5: Variation of radiation parameter $\mathrm{Nr}$ with interface heat transfer $-\theta^{\prime}(0)$ for different values of $f_{w}$.

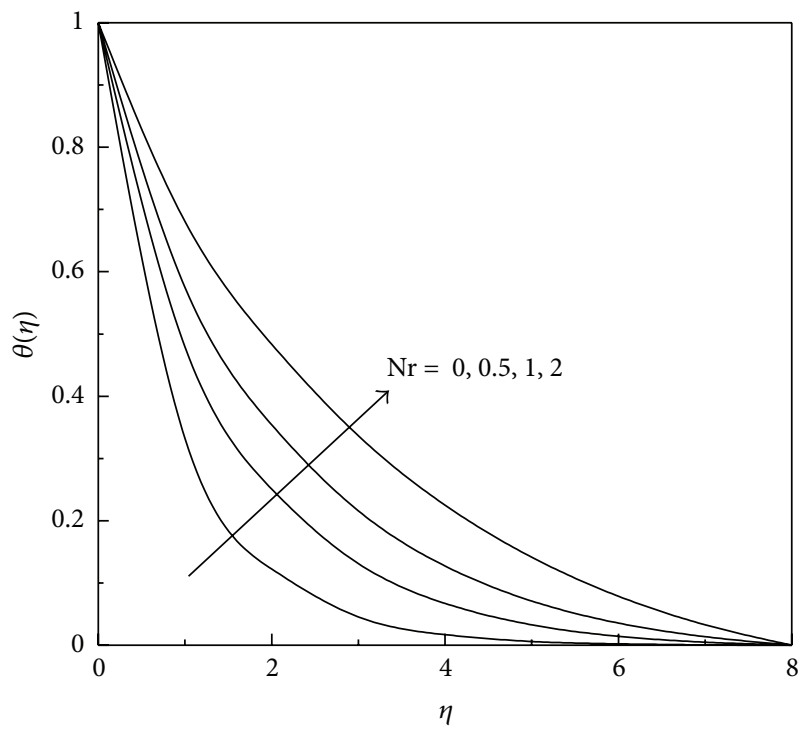

FIGURE 6: Temperature profiles for different values of Nr when $f_{w}=$ 1 .

$\mathrm{Nr}$ increases. Thus, radiation can be used to control the thermal boundary layers quite effectively.

In Figure 9 we can see the combined effects of Joule heating and viscous dissipation on heat transfer rate. When $\mathrm{Ec}=0$, there are no effects of Joule and viscous heating. It can be observed that $-\theta^{\prime}(0)$ reduced sharply with the increasing of Ec. Further, imposition of the fluid suction has the tendency to increase the interface heat transfer. On other hand, fluid injection tends to decrease the heat transfer rate at the wall but rapidly increase the rate as Ec $>3$. Meanwhile in Figures 10, 11, and 12 present the effect of the viscous dissipation on temperature profiles in the presence of suction and injection. It is noticed that increases as Ec increase the temperature.

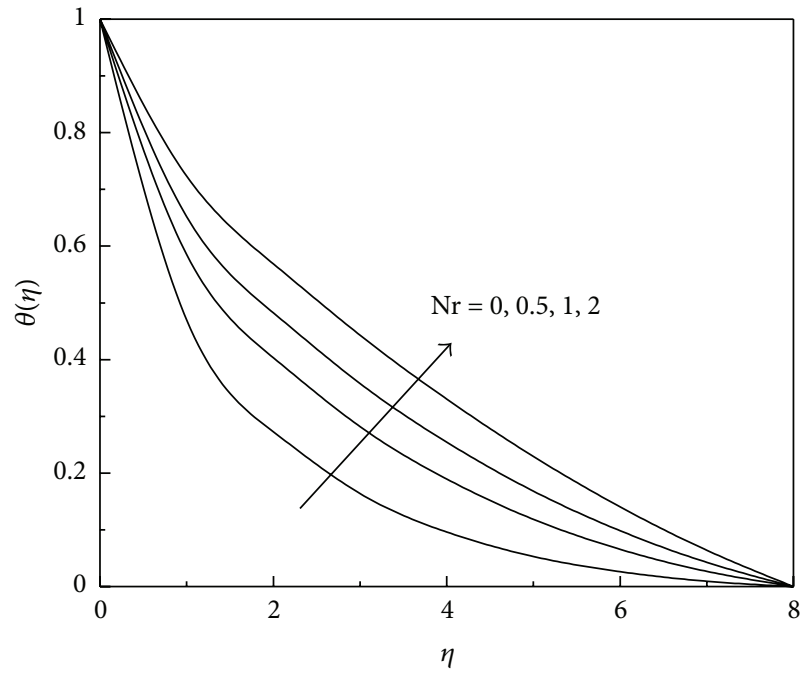

Figure 7: Temperature profiles for different values of $\operatorname{Nr}$ when $f_{w}=$ 0.

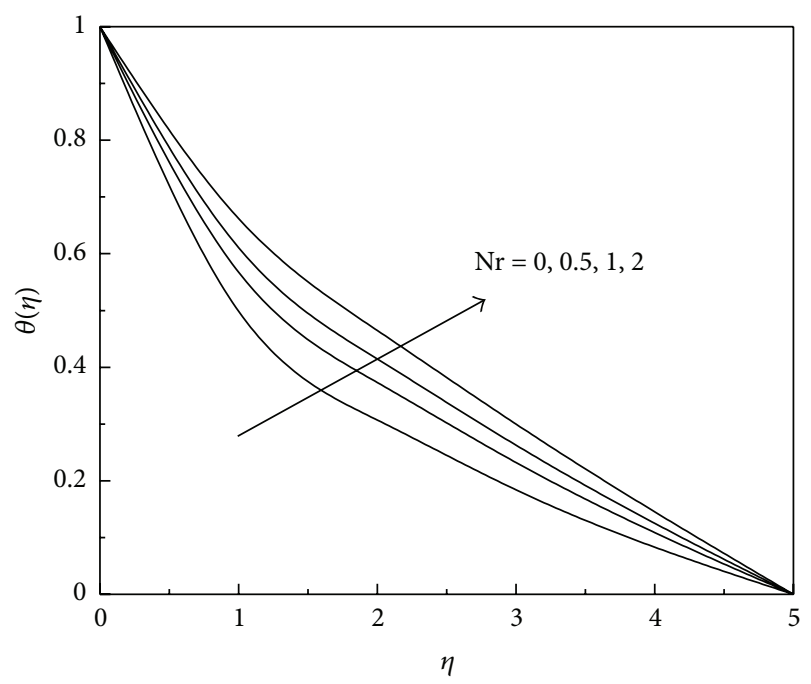

Figure 8: Temperature profiles for different values of $\operatorname{Nr}$ when $f_{w}=$ -1 .

The influence of the Schmidt number Sc on the concentration of the fluid in presence of suction or injection parameter $f_{w}$ is presented in Figures 13 and 14. Figure 13 presents the effect of Schmidt number Sc on the reduced species gradient, $-\phi^{\prime}(0)$, under the presence of different values of $f_{w}$. It is noted that fluid suction or injection has the tendency to increase the interface mass transfer. The effect of Schmidt number Sc on the concentration is presented in Figure 14. It is evident that the concentration decreases with an increase in Sc.

Figures 15, 16, and 17 illustrate the influence of the suction or injection parameter $f_{w}$ on the velocity, temperature, and concentration, respectively. Physically speaking, imposition of fluid suction $\left(f_{w}>0\right)$ at the wall has the tendency to decrease the fluid velocity and thickness of the hydrodynamic 


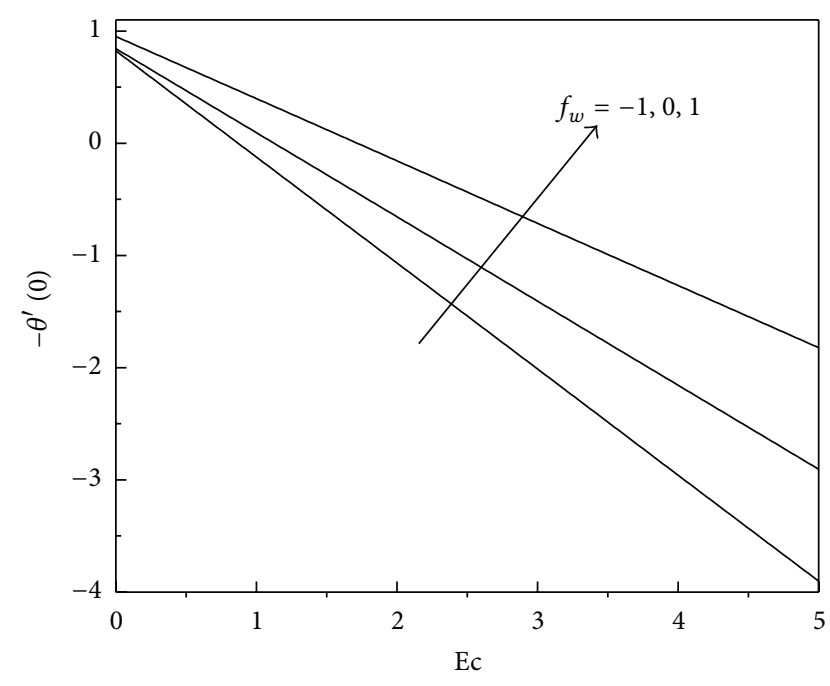

FIGURE 9: Variation of Eckert number Ec with interface heat transfer $-\theta^{\prime}(0)$ for different values of $f_{w}$.

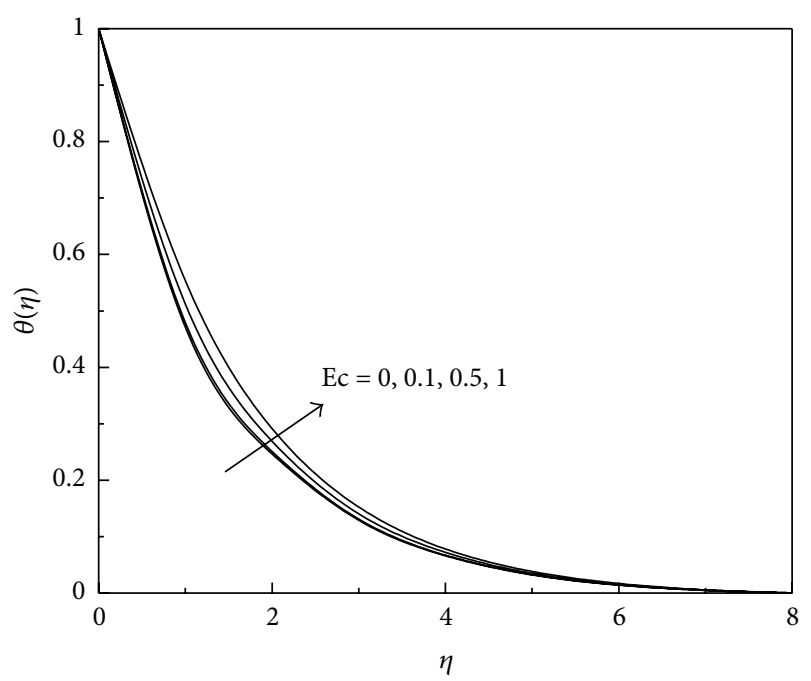

FIGURE 10: Temperature profiles for different values of Ec when $f_{w}=$ 1.

TABle 1: Comparison values of $f^{\prime}(0)$ and $-\theta^{\prime}(0)$ with different parameter $M$.

\begin{tabular}{lcccc}
\hline \multirow{2}{*}{$M$} & \multicolumn{2}{c}{ Al-Mudhaf and Chamkha [12] } & \multicolumn{2}{c}{ Present } \\
& $f^{\prime}(0)$ & $-\theta^{\prime}(0)$ & $f^{\prime}(0)$ & $-\theta^{\prime}(0)$ \\
\hline 0 & 1.587671 & 1.442203 & 1.58155 & 1.440125 \\
1 & 1.315181 & 1.206468 & 1.31379 & 1.20448 \\
2 & 0.903945 & 0.759604 & 0.90321 & 0.75752 \\
3 & 0.644888 & 0.442240 & 0.64402 & 0.44196 \\
4 & 0.493358 & 0.272847 & 0.49248 & 0.27452 \\
\hline
\end{tabular}

boundary layer. As a result, the fluid temperature and concentration boundary layer decrease as well. However, fluid injection $\left(f_{w}>0\right)$ produces the opposite effect, namely, an increase in the fluid velocity, temperature, and concentration.

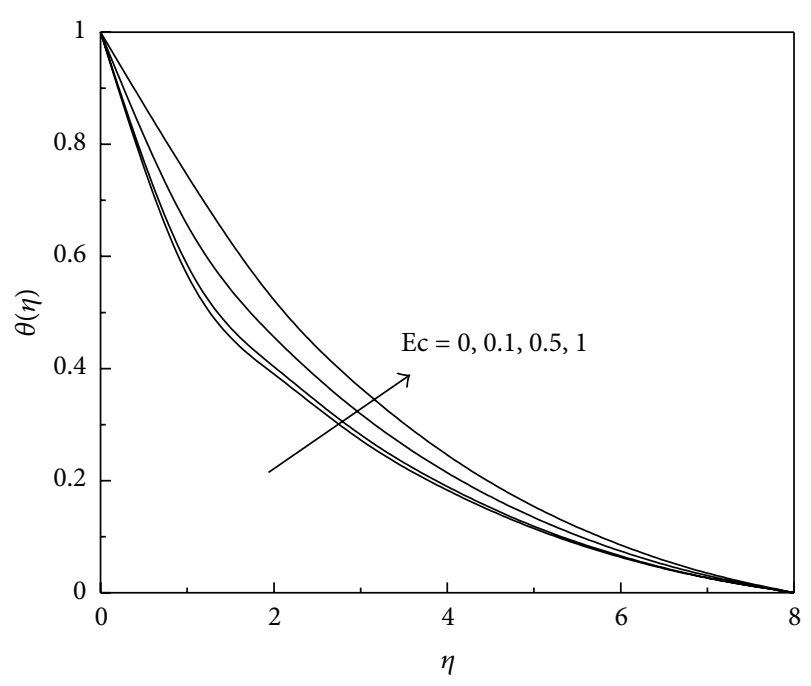

FIGURE 11: Temperature profiles for different values of Ec when $f_{w}=$ 0.

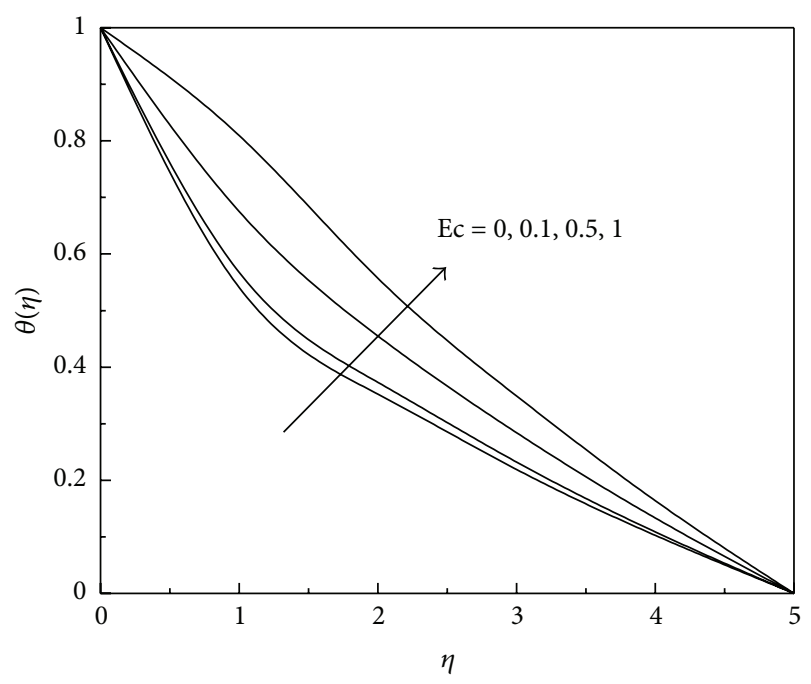

FIGURE 12: Temperature profiles for different values of Ec when $f_{w}=$ -1 .

TABle 2: Comparison values of $f^{\prime}(0)$ and $-\theta^{\prime}(0)$ with different parameter $f_{w}$.

\begin{tabular}{lcccc}
\hline \multirow{2}{*}{$f_{w}$} & \multicolumn{2}{c}{ Al-Mudhaf and Chamkha [12] } & \multicolumn{2}{c}{ Present } \\
& $f^{\prime}(0)$ & $-\theta^{\prime}(0)$ & $f^{\prime}(0)$ & $-\theta^{\prime}(0)$ \\
\hline-2 & 2.383451 & 1.251341 & 2.38963 & 1.25986 \\
-1 & 2.000379 & 1.336441 & 2.00753 & 1.32874 \\
0 & 1.587671 & 1.442203 & 1.58125 & 1.43987 \\
1 & 1.179708 & 1.634990 & 1.17324 & 1.63123 \\
2 & 0.848026 & 2.020945 & 0.83924 & 2.01785 \\
\hline
\end{tabular}

Figure 18 illustrates the variation of the surface temperature gradient $-\theta^{\prime}(0)$ with the Eckert number Ec for different values of the Prandtl number, that is, $\operatorname{Pr}=0.72,7.0$ and 10 . From Figure 18, it is observed that as Ec increases the surface 


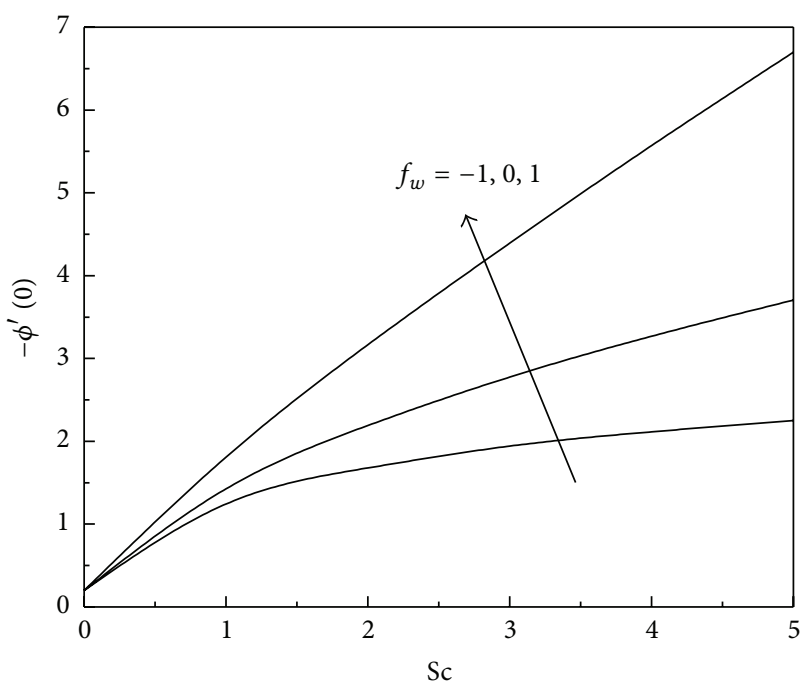

FIGURE 13: Variation of heat Schmidt number Sc with interface mass transfer $-\phi^{\prime}(0)$ for different values of $f_{w}$.

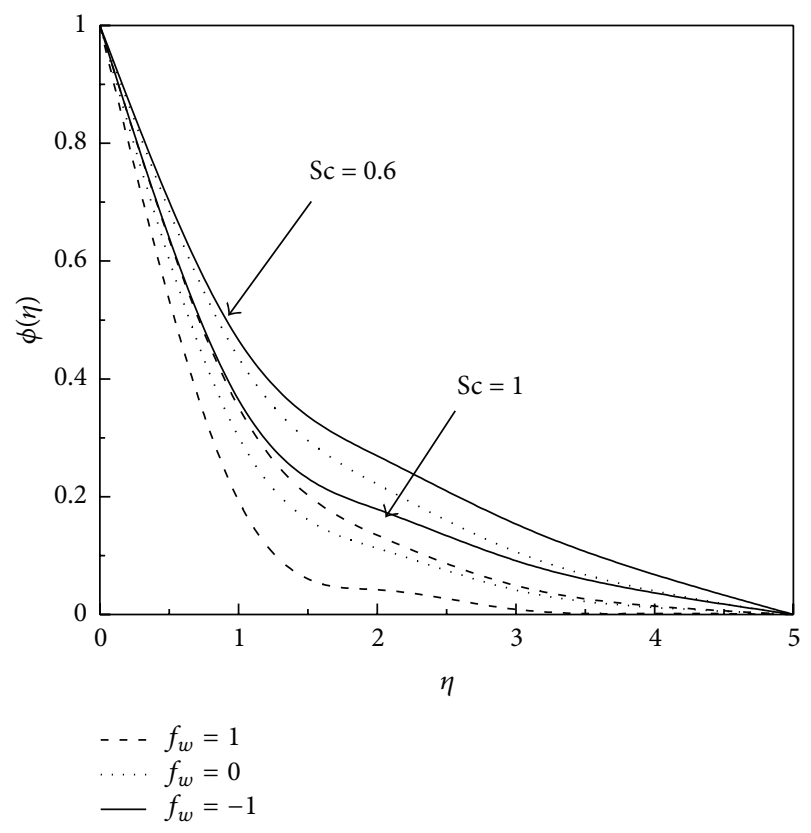

FIgURE 14: Concentration profiles for different values of Sc.

temperature gradient is found to be decreased in the presence of thermal radiation Nr. Furthermore, if we consider higher Prandtl number then we notice further reduction in the surface temperature gradient.

Tables 1 and 2 show the comparison of $f^{\prime}(0)$ and $-\theta^{\prime}(0)$ with those reported by Al-Mudhaf and Chamkha [12], which show an excellent agreement and we are confident that our present numerical results are accurate.

Further, Table 3 shows the effects of magnetic parameter $M$, Prandtl number $\mathrm{Pr}$, radiation parameter $\mathrm{Nr}$, viscous dissipation Ec, Schmidt number Sc and suction $f_{w}$ on the physical parameters surface velocity gradient $f^{\prime}(0)$, surface

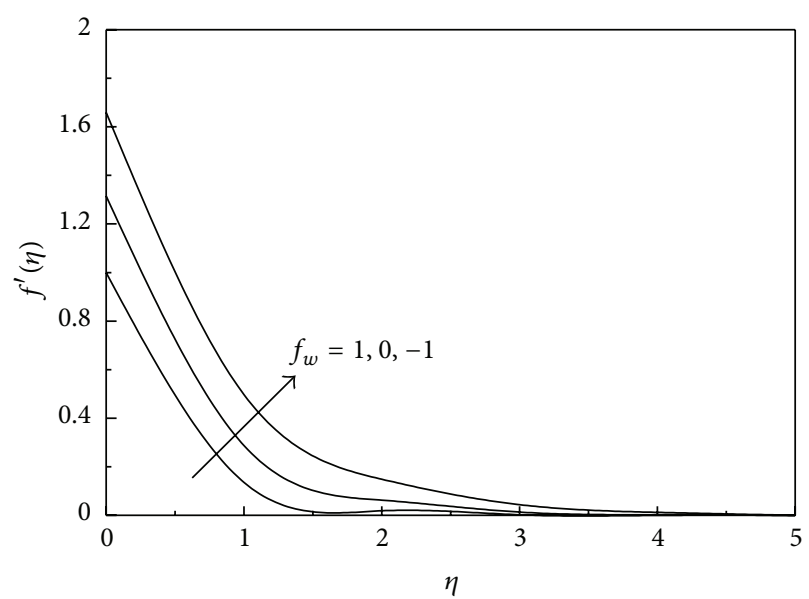

FIGURE 15: Velocity profiles for different values of $f_{w}$.

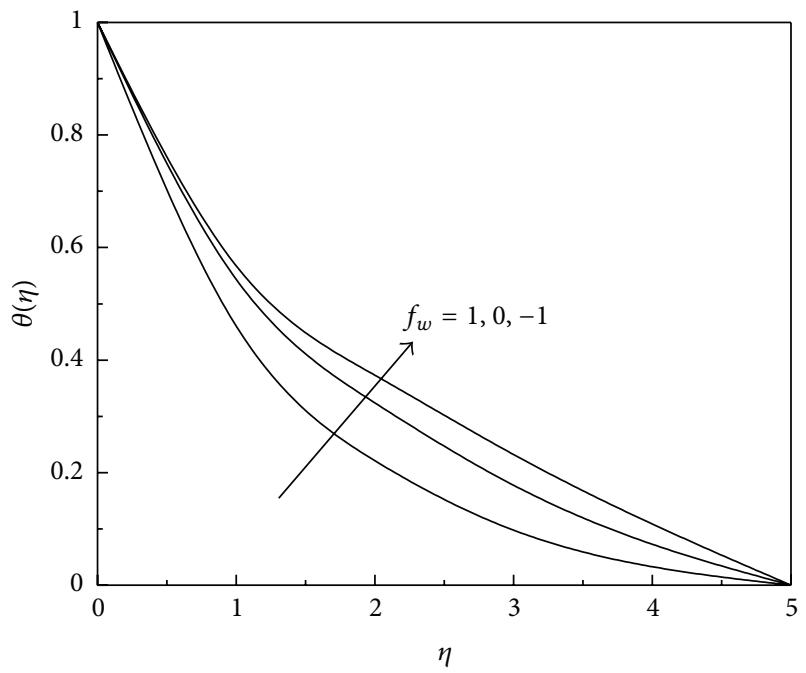

Figure 16: Temperature profiles for different values of $f_{w}$.

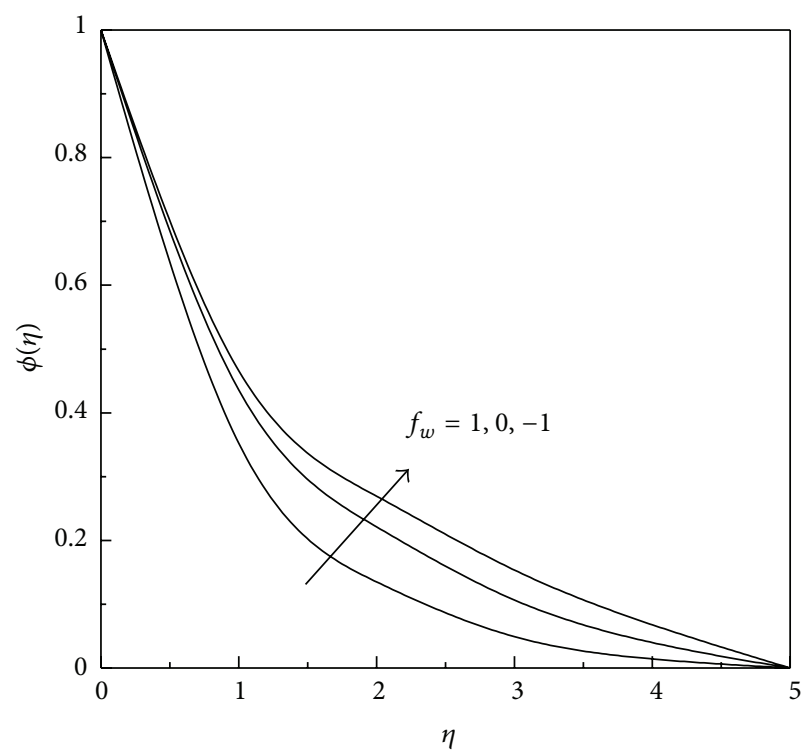

FIGURE 17: Concentration profiles for different values of $f_{w}$. 
TABLE 3: Computation showing $f^{\prime}(0),-\theta^{\prime}(0)$ and $-\phi^{\prime}(0)$ for different embedded flow parameter values.

\begin{tabular}{ccccccccc}
\hline$M$ & $\mathrm{Pr}$ & $\mathrm{Nr}$ & $\mathrm{Ec}$ & $\mathrm{Sc}$ & $f_{w}$ & $f^{\prime}(0)$ & $-\theta^{\prime}(0)$ & $-\phi^{\prime}(0)$ \\
\hline 1.0 & 0.72 & 0.5 & 0.1 & 0.6 & 1.0 & 0.999612 & 0.941925 \\
2.0 & 0.72 & 0.5 & 0.1 & 0.6 & 1.0 & 0.732048 & 0.777927 & 1.22659 \\
3.0 & 0.72 & 0.5 & 0.1 & 0.6 & 1.0 & 0.550868 & 0.683809 \\
1.0 & 1 & 0.5 & 0.1 & 0.6 & 1.0 & 0.999612 & 1.21094 & 0.915823 \\
1.0 & 2.0 & 0.5 & 0.1 & 0.6 & 1.0 & 0.999612 & 2.11956 & 1.22659 \\
1.0 & 0.72 & 1.0 & 0.1 & 0.6 & 1.0 & 0.999612 & 0.78197 & 1.22659 \\
1.0 & 0.72 & 2.0 & 0.1 & 0.6 & 1.0 & 0.999612 & 0.625854 & 1.22659 \\
1.0 & 0.72 & 0.5 & 0.2 & 0.6 & 1.0 & 0.999612 & 0.887262 & 1.22659 \\
1.0 & 0.72 & 0.5 & 0.3 & 0.6 & 1.0 & 0.999612 & 0.832598 \\
1.0 & 0.72 & 0.5 & 0.1 & 1.0 & 1.0 & 0.999612 & 0.941925 \\
1.0 & 0.72 & 0.5 & 0.1 & 1.5 & 1.0 & 0.999612 & 0.941925 \\
1.0 & 0.72 & 0.5 & 0.1 & 0.6 & 2.0 & 0.752099 & 1.19244 \\
1.0 & 0.72 & 0.5 & 0.1 & 0.6 & 3.0 & 0.578583 & 1.81808 \\
\hline
\end{tabular}

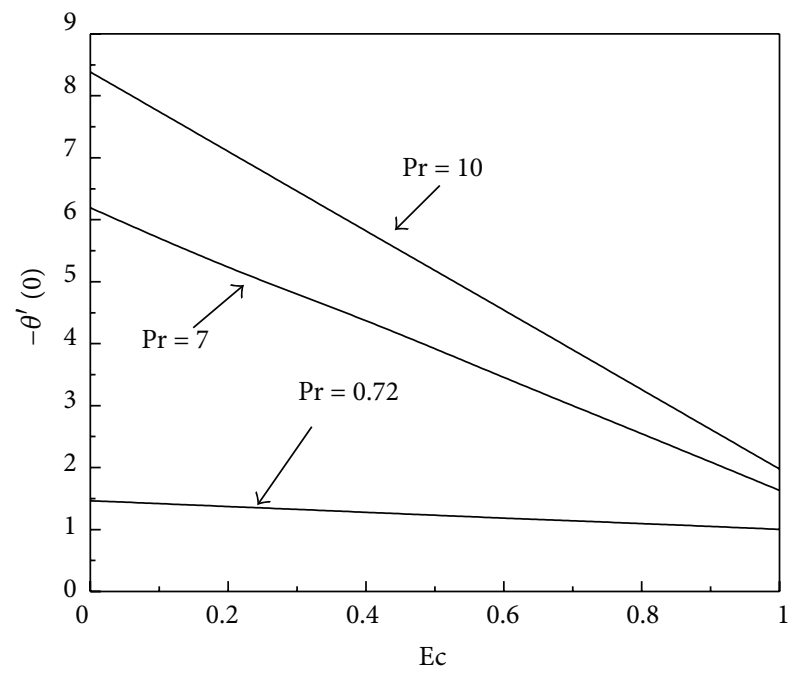

FIGURE 18: Variation of the surface temperature gradient $-\theta^{\prime}(0)$ with the Eckert number Ec for different values of Pr.

temperature gradient $-\theta^{\prime}(0)$ and surface concentration gradient $-\phi^{\prime}(0)$, respectively. It can be seen that all of $f^{\prime}(0)$, $-\theta^{\prime}(0)$, and $-\phi^{\prime}(0)$ decrease as magnetic field parameter $M$ increases. $-\theta^{\prime}(0)$ increases as Prandtl number Pr increases. $-\theta^{\prime}(0)$ decreases as radiation $\mathrm{Nr}$ and viscous dissipation Ec increase. $-\phi^{\prime}(0)$ decreases as Schmidt number Sc increase and as suction parameter $f_{w}$ increases $f^{\prime}(0)$ decreases while $-\theta^{\prime}(0)$ and $-\phi^{\prime}(0)$ increase.

\section{Conclusions}

The combined effects of mass transfer and thermal radiation on a steady laminar MHD Marangoni convection boundary layer flow over a flat surface by taking the Joule heating and viscous dissipation in the influence of fluid suction and injection. The governing partial differential equations are reduced to a system of self-similar equations using the similarity transformations. The resultant equations are then solved numerically using the Runge-Kutta method along with shooting technique. Comparison with previously published work was performed and the results were found to be in excellent agreement. The effects of governing physical parameters on the velocity, temperature, and concentration as well as surface temperature gradient and surface concentration are computed and presented in graphical and tabular forms. It was found that the temperature increases as Eckert number $\mathrm{Ec}$ or radiation parameter $\mathrm{Nr}$ increases, while temperature decreases as Prandtl number Pr or magnetic field $M$ increases, whereas concentration decreases as Schmidt number Sc increases. It can be drawn from the present results that when the radiation parameter increases, the heat transfer rate at the surface decreases. Meanwhile, the imposition of suction is to decrease the fluid velocity, temperature, and concentration profiles, whereas injection shows the opposite effects. It should be noted that results obtained in this work can used for the analysis of Marangoni flow and heat and mass transfer for flow over curved surfaces provided that the curvature is much greater than the boundary layer thickness.

\section{Conflict of Interests}

Dr. S. Mohammed Ibrahim, the author of this paper declares that there is no conflict of interests regarding the publication of this paper.

\section{References}

[1] K. Nishino and H. Kawamura, Chaos, Turbulence and Its Transition Process in Marangoni Convection, KIBO Japanese Experiment Module, Japan Aerospace Exporation Agency, 2007.

[2] K. Arafune and A. Hirata, "Thermal and solutal marangoni convection in In-Ga-Sb system," Journal of Crystal Growth, vol. 197, no. 4, pp. 811-817, 1999.

[3] K. Arafune, K. Yamamoto, and A. Hirata, "Interactive thermal and solutal Marangoni convection during compound semiconductor growth in a rectangular open boat," International Journal of Heat and Mass Transfer, vol. 44, no. 13, pp. 2404-2411, 2001. 
[4] Z. Galazka and H. Wilke, "Influence of Marangoni convection on the flow pattern in the melt during growth of $\mathrm{Y}_{3} \mathrm{Al}_{5} \mathrm{O}_{12}$ single crystals by the Czochralski method," Journal of Crystal Growth, vol. 216, no. 1, pp. 389-398, 2000.

[5] H. Neumann, Y. Plevachuk, and F. Allenstein, "Investigation of Marangoni convection in monotectic melts by resistance measurements," Materials Science and Engineering A, vol. 361, no. 1-2, pp. 155-164, 2003.

[6] B. Arendt and R. Eggers, "Interaction of Marangoni convection with mass transfer effects at droplets," International Journal of Heat and Mass Transfer, vol. 50, no. 13-14, pp. 2805-2815, 2007.

[7] Y. L. Xu, Z. B. Dong, Y. H. Wei, and C. L. Yang, "Marangoni convection and weld shape variation in A-TIG welding process," Theoretical and Applied Fracture Mechanics, vol. 48, no. 2, pp. 178-186, 2007.

[8] D. M. Christopher and B. Wang, "Prandtl number effects for Marangoni convection over a flat surface," International Journal of Thermal Sciences, vol. 40, no. 6, pp. 564-570, 2001.

[9] Y. Jaluria, Natural Convection, Heat and Mass Transfer, Pergamon Press, New York, NY, USA, 1980.

[10] M. Itaru, T. Osamu, and S. Akimi, "Natural convection heat transfer from a horizontal cylinder to mercury under magnetic field," International Journal of Heat and Mass Transfer, vol. 19, no. 9, pp. 1021-1029, 1976.

[11] M.-O. Fumizawa, "Natural convection experiment with liquid nak under transverse magnetic field," Journal of Nuclear Science and Technology, vol. 17, no. 2, pp. 98-105, 1980.

[12] A. Al-Mudhaf and A. J. Chamkha, "Similarity solutions for MHD thermosolutal Marangoni convection over a flat surface in the presence of heat generation or absorption effects," Heat and Mass Transfer/Waerme- und Stoffuebertragung, vol. 42, no. 2, pp. 112-121, 2005.

[13] G. Pathak and C. H. Maheshwari, "Effect of radiation on unsteady free convection flow bounded by an oscillating plate with variable wall temperature," International Journal of Applied Mechanics and Engineering, vol. 11, no. 2, pp. 371-382, 2006.

[14] S. Shateyi, "Thermal radiation and buoyancy effects on heat and mass transfer over a semi-infinite stretching surface with suction and blowing," Journal of Applied Mathematics, vol. 2008, Article ID 414830, 12 pages, 2008.

[15] S. Suneetha, N. B. Reddy, and V. R. Prasad, "Thermal radiation effects on MHD free convection flow past an impulsively started vertical plate with variable surface temperature and concentration," Journal of Naval Architecture and Marine Engineering, vol. 2, pp. 57-70, 2008.

[16] M. A. Alim, M. D. Alam, and A. Mamun, "Joule heating effect on the coupling of conduction with magnetohydrodynamic free convection flow from a vertical plate," Nonlinear Analysis Modelling and Control, vol. 12, no. 3, pp. 307-316, 2007.

[17] V. D. Borisevich and E. P. Potanin, "Effects of viscous dissipating and Joule heat on heat transfer near a rotating disk in the presence of intensive suction," Journal of Engineering Physics, vol. 55, no. 5, pp. 1220-1223, 1988.

[18] H. M. Duwairi, "Viscous and Joule heating effects on forced convection flow from radiate isothermal porous surfaces," International Journal of Numerical Methods for Heat and Fluid Flow, vol. 15, no. 5, pp. 429-440, 2005.

[19] C.-H. Chen, "Combined effects of Joule heating and viscous dissipation on Magnetohydrodynamic flow past a permeable, stretching surface with free convection and radiative heat transfer," Journal of Heat Transfer, vol. 132, no. 6, pp. 1-5, 2010.
[20] T. S. Khaleque and M. A. Samad, "Effects of radiation, heat generation and viscous dissipation on MHD free convection flow along a stretching sheet," Research Journal of Applied Sciences, Engineering and Technology, vol. 2, no. 4, pp. 368-377, 2010.

[21] M. Q. Brewster, Thermal Radiative Transfer Properties, John Wiley \& Sons, New York, NY, USA, 1992.

[22] A. Ishak, "Radiation effects on the flow and heat transfer over a moving plate in a parallel stream," Chinese Physics Letters, vol. 26, no. 3, pp. 1-4, 2009. 


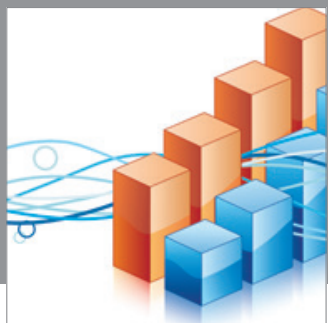

Advances in

Operations Research

mansans

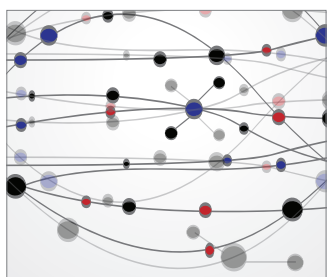

The Scientific World Journal
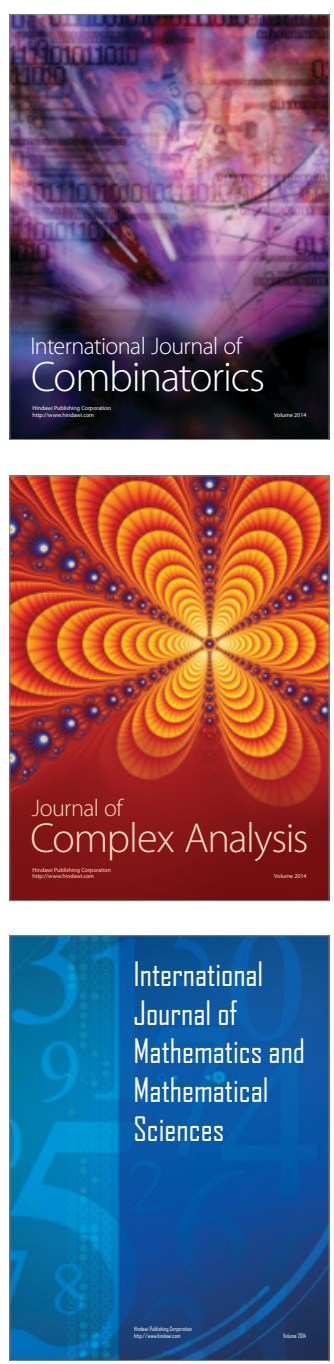
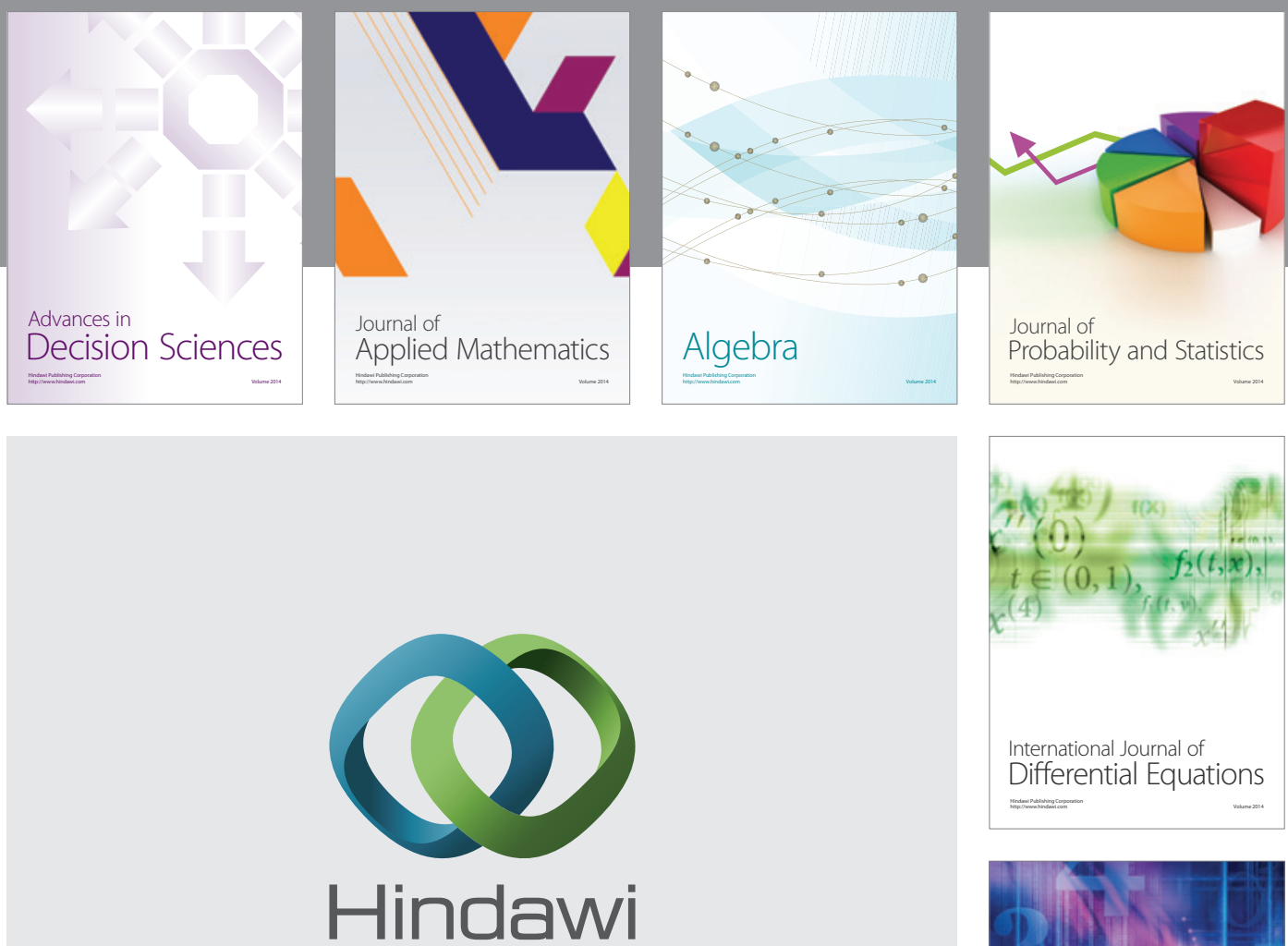

Submit your manuscripts at http://www.hindawi.com
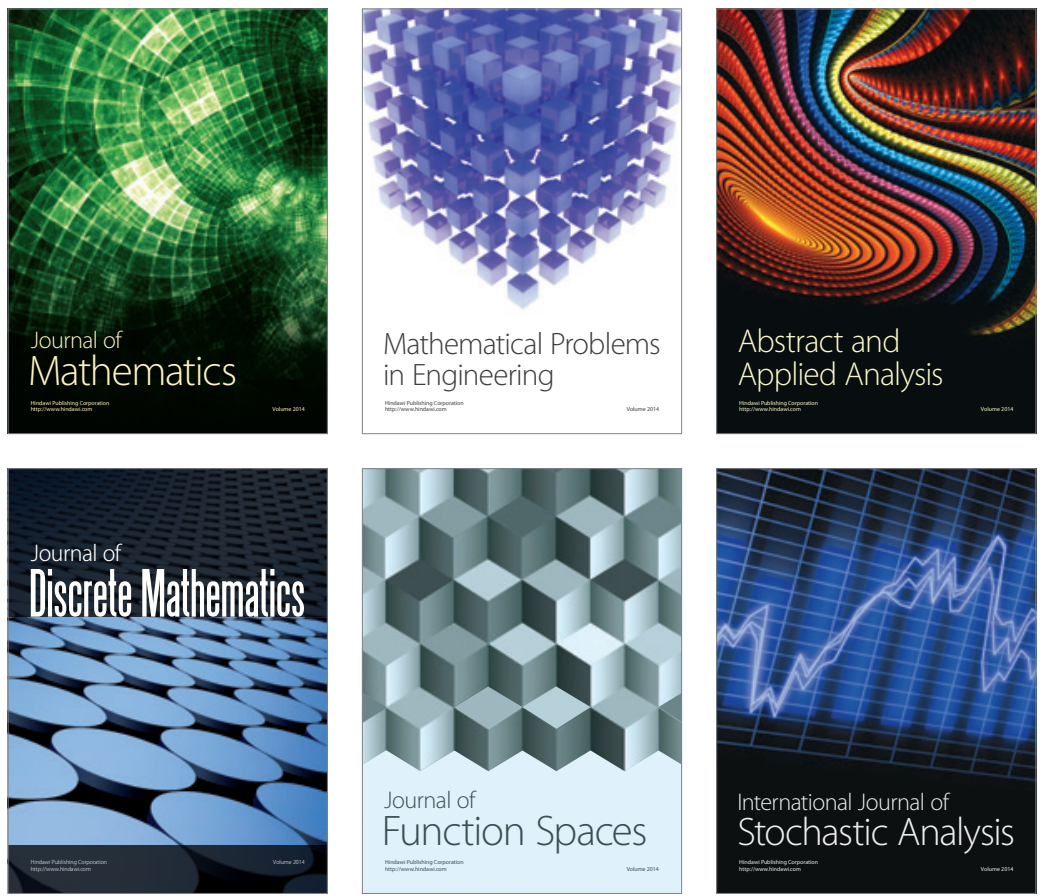

Journal of

Function Spaces

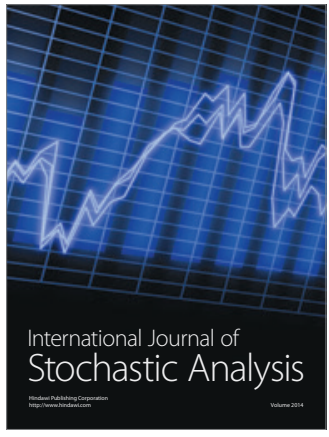

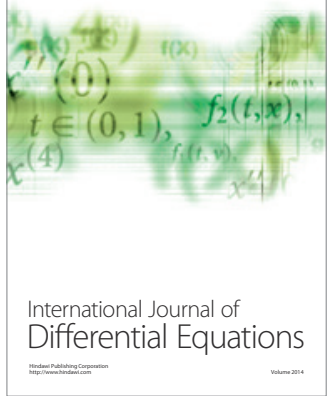
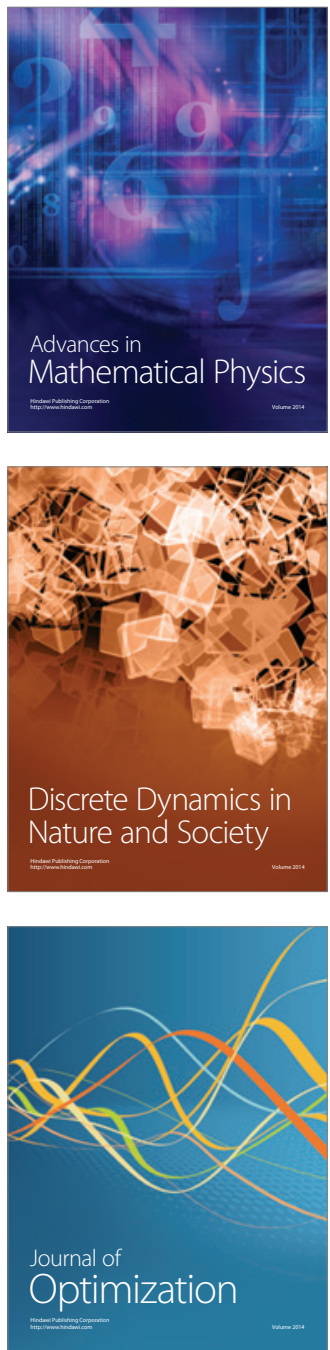